\title{
Article \\ Optimization of the In Situ Biasing FIB Sample Preparation for Hafnia-Based Ferroelectric Capacitor
}

\author{
Qilan Zhong ${ }^{1,+}$, Yiwei Wang ${ }^{1,+}{ }^{1}$, Yan Cheng ${ }^{1, *}$, Zhaomeng Gao ${ }^{2,3}$, Yunzhe Zheng ${ }^{1}$, Tianjiao Xin ${ }^{1}$, \\ Yonghui Zheng ${ }^{1}$, Rong Huang ${ }^{1}$ and Hangbing Lyu ${ }^{2,3, *}$ \\ 1 Key Laboratory of Polar Materials and Devices (MOE), Department of Electronics, \\ East China Normal University, 500 Dong-chuan Road, Shanghai 200241, China; \\ 52191213009@stu.ecnu.edu.cn (Q.Z.); 51214700096@stu.ecnu.edu.cn (Y.W.); \\ 52181213007@stu.ecnu.edu.cn (Y.Z.); 52214700045@stu.ecnu.edu.cn (T.X.); yhzheng@phy.ecnu.edu.cn (Y.Z.); \\ rhuang@ee.ecnu.edu.cn (R.H.) \\ 2 Key Laboratory of Microelectronics Devices and Integrated Technology, Institute of Microelectronics, \\ Chinese Academy of Sciences, 3 Bei-tu-cheng West Road, Beijing 100029, China; gaozhaomeng@ime.ac.cn \\ 3 University of Chinese Academy of Sciences, Beijing 100049, China \\ * Correspondence: ycheng@ee.ecnu.edu.cn (Y.C.); lvhangbing@ime.ac.cn (H.L.); Tel.: +86-21-5434-5398 (Y.C.) \\ + These authors contributed equally.
}

Citation: Zhong, Q.; Wang, Y.;

Cheng, Y.; Gao, Z.; Zheng, Y.; Xin, T.;

Zheng, Y.; Huang, R.; Lyu, H.

Optimization of the In Situ Biasing FIB Sample Preparation for Hafnia-Based Ferroelectric Capacitor. Micromachines 2021, 12, 1436.

https://doi.org/10.3390/mi12121436

Academic Editor: Rosa Córdoba

Received: 27 October 2021

Accepted: 21 November 2021

Published: 24 November 2021

Publisher's Note: MDPI stays neutral with regard to jurisdictional claims in published maps and institutional affiliations.

Copyright: (c) 2021 by the authors. Licensee MDPI, Basel, Switzerland. This article is an open access article distributed under the terms and conditions of the Creative Commons Attribution (CC BY) license (https:// creativecommons.org/licenses/by/ $4.0 /)$.

\begin{abstract}
Hafnia-based ferroelectric (FE) thin films have received extensive attention in both academia and industry, benefitting from their outstanding scalability and excellent CMOS compatibility. Hafniabased FE capacitors in particular have the potential to be used in dynamic random-access memory (DRAM) applications. Obtaining fine structure characterization at ultra-high spatial resolution is helpful for device performance optimization. Hence, sample preparation by the focused ion beam (FIB) system is an essential step, especially for in situ biasing experiments in a transmission electron microscope (TEM). In this work, we put forward three tips to improve the success rate of in situ biasing experiments: depositing a carbon protective layer to position the interface, welding the sample on the top of the $\mathrm{Cu}$ column of the TEM grid, and cutting the sample into a comb-like shape. By these means, in situ biasing of the FE capacitor was realized in TEM, and electric-field-induced tetragonal ( $\mathrm{t}-$ ) to monoclinic (m-) structure transitions in $\mathrm{Hf}_{0.5} \mathrm{Zr}_{0.5} \mathrm{O}_{2} \mathrm{FE}$ film were observed. The improvement of FIB sample preparation technology can greatly enhance the quality of in situ biasing TEM samples, improve the success rate, and extend from capacitor sample preparation to other types.
\end{abstract}

Keywords: hafnia-based ferroelectric; in situ biasing; FIB sample preparation technology

\section{Introduction}

Since the discovery of the ferroelectric properties of hafnia-based thin film, there has been significant interest in both its fundamentals and application. Ferroelectric thin films based on $\mathrm{HfO}_{2}$ enable new types of computer memory and logic devices, such as ultra-low power transistors, artificial synapse tunable tunnel barriers, and non-volatile working memory [1-5]. However, $\mathrm{HfO}_{2}$-based ferroelectric thin films have a variety of crystalline phases, including monoclinic $\left(\mathrm{m}-, \mathrm{P} 2{ }_{1} / \mathrm{c}\right)$, tetragonal $\left(\mathrm{t}-, \mathrm{P} 4_{2} / \mathrm{nmc}\right)$, cubic (c-, Fm $3 \bar{m}$ ), and orthorhombic (o-, Pca2 ${ }_{1}, \mathrm{Pbca}$ and Pnma) phases [6,7]. The accompanying structural phase transition is very complex. The possible transition paths from t-phase to non-centrosymmetric ferroelectric o-phase and from t-phase to $\mathrm{m}$-phase have been predicted by theoretical calculation on the basis of considering surface/interface energy, stress/strain and electric field [8-11]. Understanding the structural phase transition of ferroelectric thin films under electric field, thermal field, and other external field conditions is a research hotspot [12,13].

Transmission electron microscopes (TEM), as advanced equipment, make it possible to identify different phases and structures of hafnia-based materials at the atomic 
scale [14-17]. In recent years, a variety of in situ techniques combined with TEM have expanded the analysis field and research content of electron microscopy with the characteristics of high spatial resolution and real-time recording and have become a new direction of TEM development [18-23]. In situ technology could provide various external fields to investigate the performance of devices by using different TEM sample holders. They are mainly divided into two kinds: in situ sample stations driven by piezoelectric ceramics and sample stations based on a micro-electro-mechanical system (MEMS) chip [18,24,25]. These two devices are inseparable from the support of focused ion beam (FIB) sample preparation technology; mature and excellent FIB sample preparation technology is the basis of successful in situ experiments.

Based on the piezoelectric-ceramics-type holder, we made some optimizations for sample preparation, making the sample more suitable for in situ biasing experiments. In this study, we proposed three tips to improve the success rate of in situ electrical samples by using FIB sample preparation technology. Using $\mathrm{Hf}_{0.5} \mathrm{Zr}_{0.5} \mathrm{O}_{2}$ (HZO) FE grown by highmaturity atomic layer deposition (ALD) as a model, the dynamic evolution of atomic-scale resolution under electric field conditions was successfully obtained by in situ biasing with the spherical aberration-corrected (Cs) TEM technique.

\section{Materials and Methods}

For TEM in situ observation, we made a tiny fine structure sample mounted on a $\mathrm{Cu}$ grid based on the traditional TEM sample preparation and FIB system (Helios G4, Thermo Fisher Scientific, Waltham, MA, USA). A TiN/HZO $(5 \mathrm{~nm}) / \mathrm{Nb}: \mathrm{SrTiO}_{3}$ capacitor was prepared by ALD and then rapidly annealed at $500{ }^{\circ} \mathrm{C}$ for $30 \mathrm{~s}$ in $\mathrm{N}_{2}$ atmosphere. The P-V loop measurements were performed by a semiconductor device analyzer (WGFMU, B1500A, Agilent Technologies, Santa Clara, CA, USA). The in situ experiment was carried out on a JEM Grand ARM300F microscope (JEOL, Tokyo, Japan) operated at $300 \mathrm{kV}$ with an image corrector using the PicoFemto in situ electrical holder.

\section{Results}

\subsection{The Process of In Situ TEM Sample Preparation}

Figure 1 shows the whole process of in situ TEM sample preparation using an FIB system. The sample preparation process can be summarized into five steps, which are depositing protective layers, digging pits, extracting the sample, welding the sample on $\mathrm{Cu}$ grid, and thinning. Figure 1a shows the full top view of the hafnia-based ferroelectric capacitors with different size of the electrodes. Figure $1 b, c$ show the process of depositing protective layers, which can be divided into protective-layer deposition under electron beam (EB) and under ion beam (IB). The main purpose of an EB protective layer is to protect the sample from damage under IB sputtering. If necessary, a sputtering system also can be used for protection before FIB sample preparation. Figure 1d,e show the digging process, Figure 1f,g show the sample extraction process using easy lift, Figure 1h,i show the sample welding process, and finally, Figure $1 \mathrm{j}, 1$ show the thinning process. In particular, we put forward three tips to improve the success rate of in situ experiments: 1 . The first protective layer deposits carbon for identifying the interface of sample and protective layer accurately. 2. Welding the sample on the top of the column of the $\mathrm{Cu}$ grid; 3 . Cutting the sample into comb-like shape when thinning. We will cover each tip in detail below. 

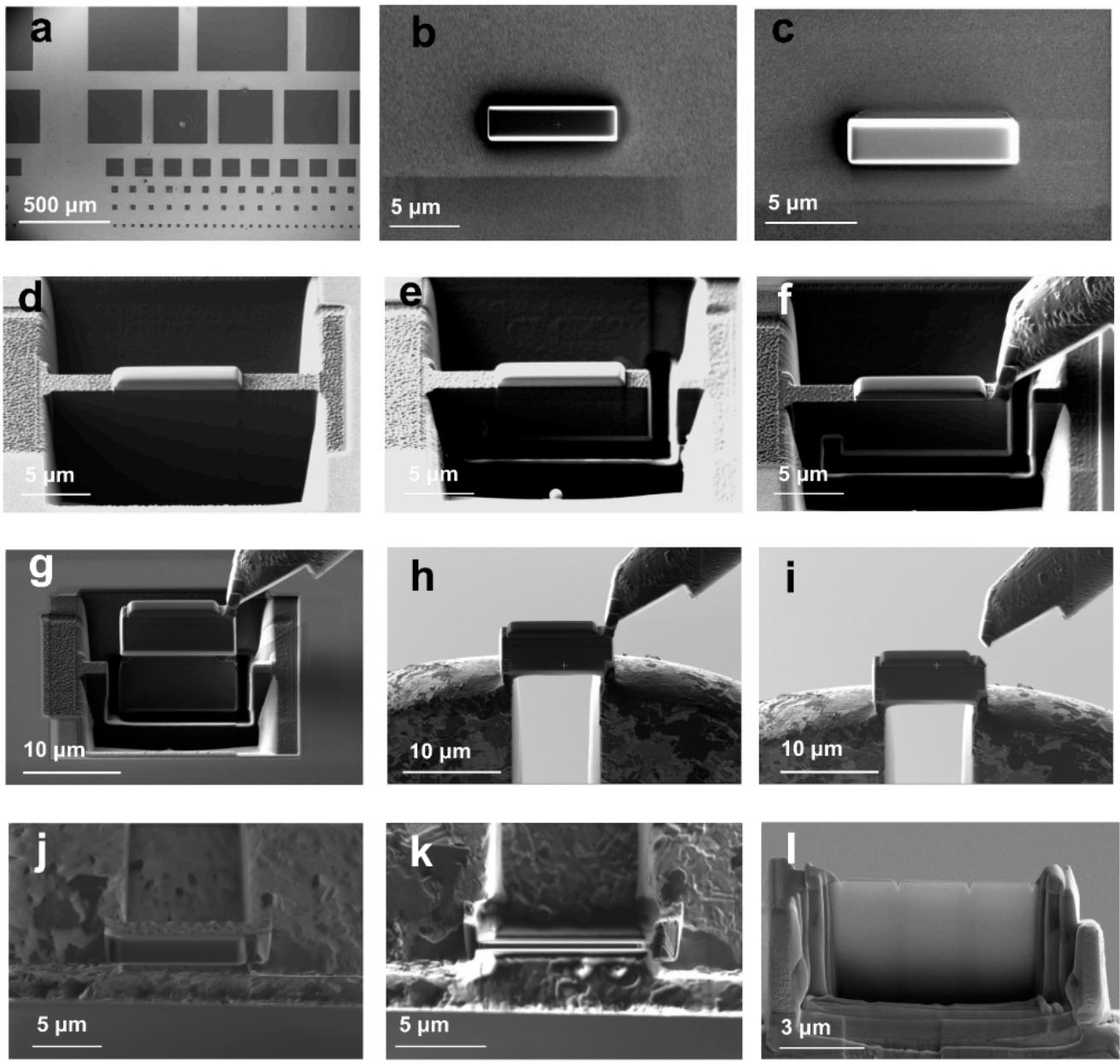

Figure 1. Focused ion beam (FIB) sample preparation step diagram. (a) Full top view of the capacitors with different size of the electrodes. (b) Deposited carbon as protective layer. (c) Deposited tungsten as protective layer. (d) Dig holes on both sides. (e) U-cut. (f) Weld easy lift to capacitor slice. (g) Lift out capacitor slice. (h) Weld capacitor slice on the Cu grid. (i) Cut the connection. (j) Cut under high current condition. (k) Cut under low current condition. (1) Cut into three comb-like capacitor slices.

\subsection{Depositing a Carbon Protective Layer to Position the Interface}

Figure 2 shows the comparison between the use of a carbon protective layer as a marker layer and another protective layer. As shown in Figure 2a and enlarged images in Figure $2 b$, when the first protective layer on the sample surface is carbon, it can be used as an accurate positioning basis. Because carbon has a lower atomic number, it exhibits a darker contrast under a slightly higher voltage of the scanning electron microscope (SEM). Accurate positioning can optimize the thinning effect, thus ensuring the imaging quality of the observed area and the best electrification effect in the experiment. The carbon protective layer can be taken many forms, such as a first coating, including direct deposition using a sputtering system, deposition under EB in a dual-beam system, and even direct deposition using a marker pen. If tungsten alone is used as a protective coating, the tungsten layer will show different contrast under different EB and IB condition, and then, it is difficult to distinguish the interface position of different protective layers and the sample, as shown in Figure 2c,d. Especially for capacitor samples with multilayer films, it is not clear where the sample is, and this problem also exists with the use of platinum as a protective layer. 

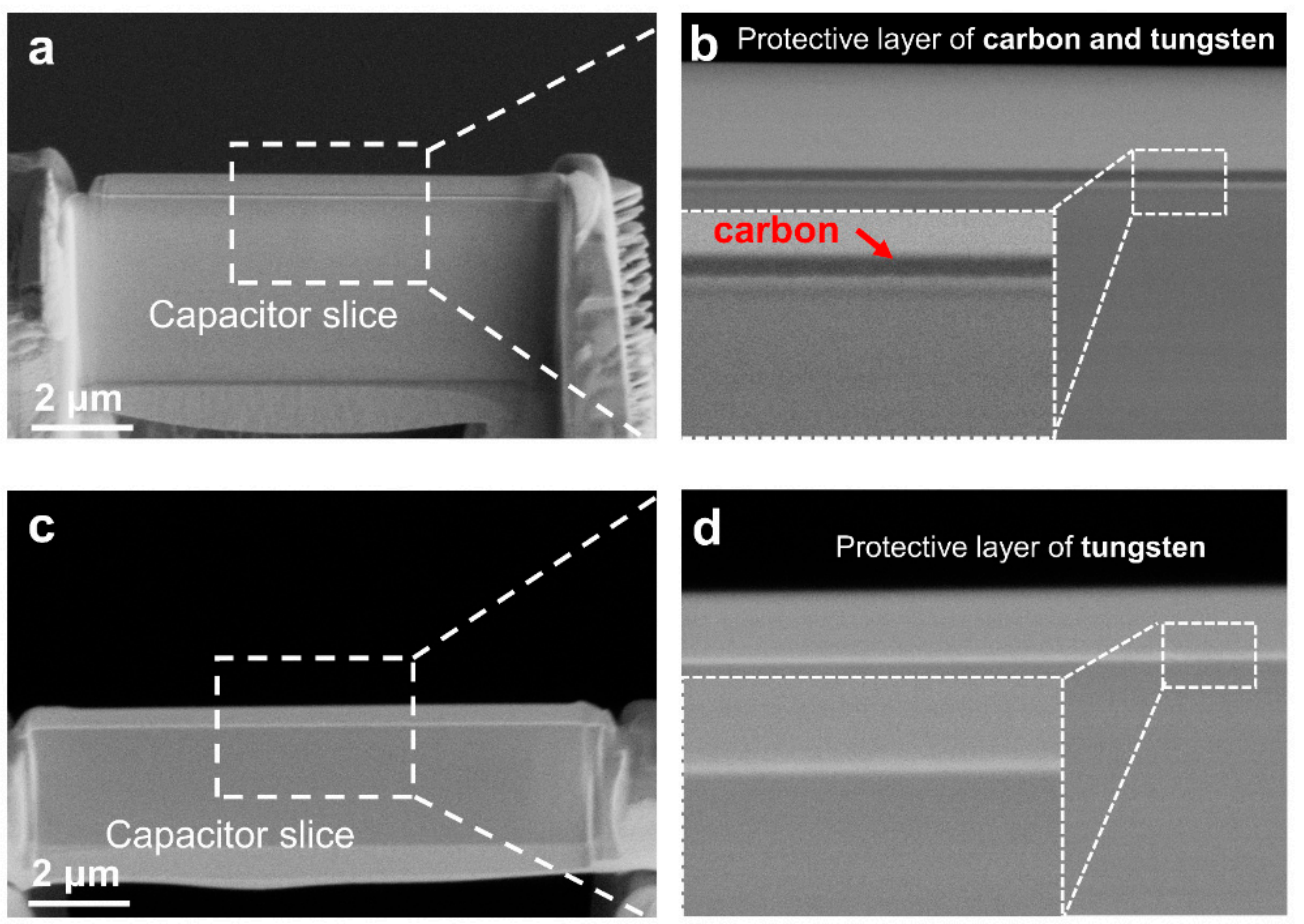

Figure 2. Comparison of different protective layers in thinning. (a) The sample is first covered with a protective carbon layer. (b) Magnified partial sample of a. (c) The sample is covered with the protective tungsten layers. (d) Magnified partial sample of (c).

\subsection{Welding the Sample on the Top of the Column of the $\mathrm{Cu}$ Grid}

Generally, the shape of copper grid is columnar and V-shaped. If it is common TEM sample preparation, the sample can be welded at any position. For the sample used in the in situ experiment of the piezoceramic driven, we propose that it can be welded at the top of the column. As shown in Figure 3, welding the sample at the top of the column has two advantages. Firstly, as shown in Figure 3a, the sample thinned with FIB can be further thinned with a Precision Ion Polishing System (PIPS) to reduce the film thickness and improve the observation quality. If the sample is welded on the side of the column, the Ar ion beam will be shielded by the grid column during PIPS thinning, while the sample welded on the top can be thinned and polished in a more comprehensive way. Secondly, Figure $3 c, d$ are enlarged images of the regions A and B in Figure 3b, respectively. We simulated the contact position of the in situ probe on the sample. As shown in Figure 3c, during the in situ experiment, the probe range was not affected, and the tungsten probe could contact a wide range of activities. However, when the sample was welded to the side of the column, it was found that the range of activity of the tungsten probe was severely limited, and the large area on the left side of the sample could hardly contact the tungsten probe for experiment. In particular, for polycrystalline samples such as hafniumbased ferroelectric films, different regions have different crystal phases, so a large range of experimental scope is very important to ensure that regions of interest can be found. Hence, we propose to weld the sample on the top of the column, which is more conducive to the in situ electrical experiment. Even in normal transmission sample preparation, it is recommended to weld the sample to the top of the column for further polishing and optimization of the sample. 

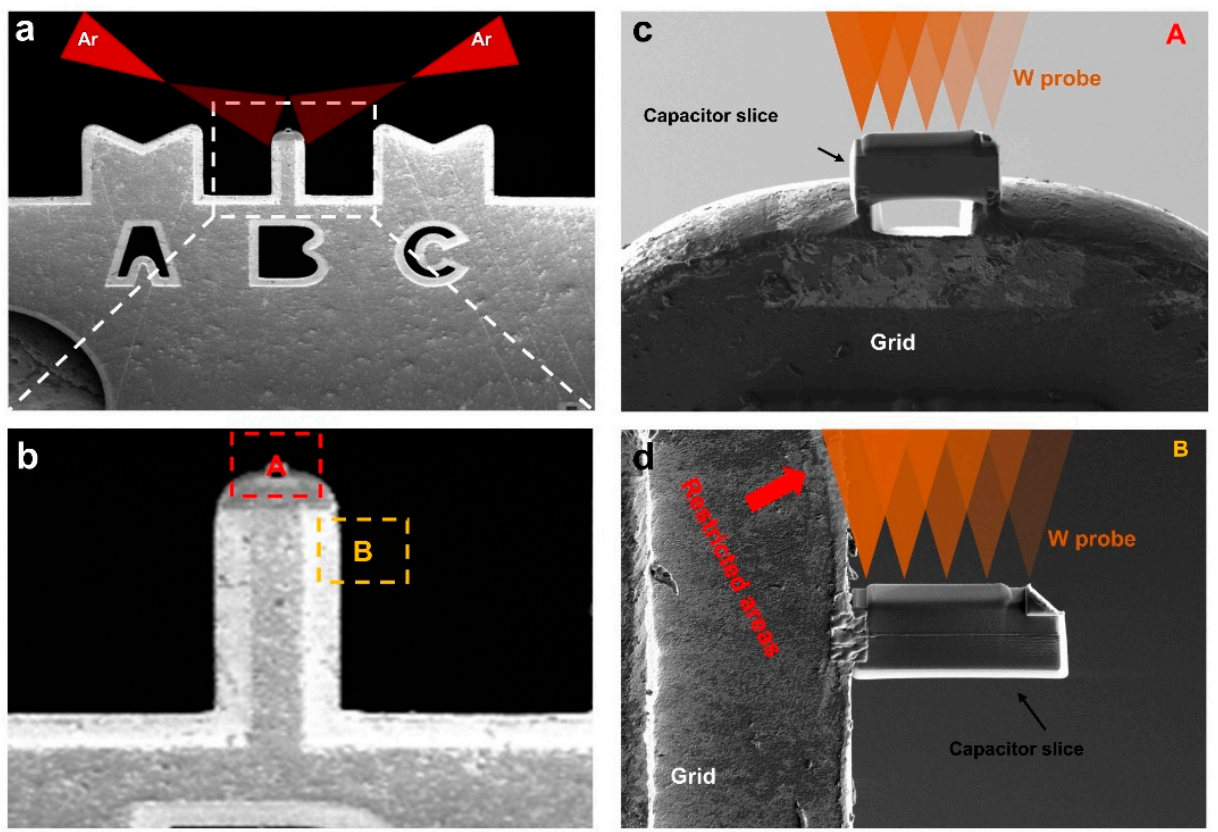

Figure 3. Advantages of welding the sample to the top of the column. (a) Simulation of polishing sample in Precision Ion Polishing System (PIPS). (b) Magnified column section of Cu grid. (c) The case that sample welded at the top of the column. (d) The case that sample welded at the side of the column.

\subsection{Cutting the Sample into "Comb" Shape When Thinning}

Bad contact and discharge are two main causes of failure of in situ electrical experiments. In order to minimize the experimental failure rate, we cut the sample into the shape of a comb during the final thinning, as shown in Figure 4. The capacitor chip was divided into several small capacitors, which were divided into three small capacitor structures, as shown in the figure. Where the tungsten probe pointed to the capacitor area, only the capacitor in this area would be conductive. The schematic diagram in the figure shows that only Capacitor 2 would be conductive, and only the blue area was conductive. In this way, the risk of short circuit and leakage can be reduced as much as possible, and the success rate of in situ electrical experiment can be guaranteed.

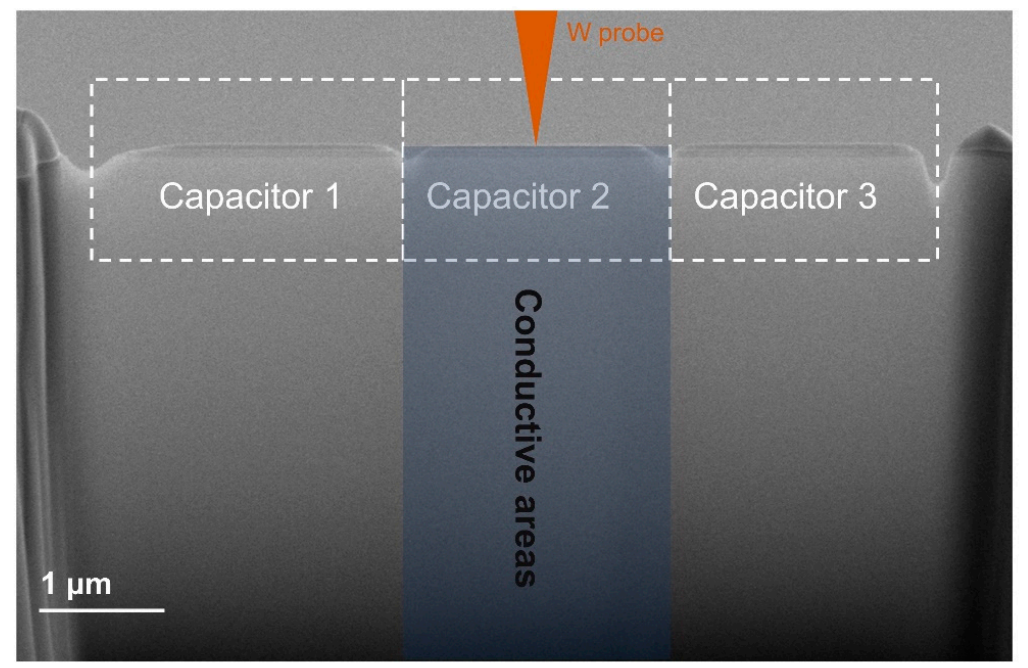

Figure 4. Final view of the comb-like capacitors for in situ electrical and structural characterization in transmission electron microscopy (TEM). 


\subsection{In Situ Biasing Experiment of Structural Transformation in Hafnia-Based Ferroelectric Thin Film}

Based on the above three tips, we successfully prepared a hafnia-based ferroelectric capacitor sample for in situ electrical experiments. The sample structure is shown in Figure 5a; the hafnia-based film was a 1:1 mixture of $\mathrm{HfO}_{2}$ and $\mathrm{ZrO}_{2}\left(\mathrm{Hf}_{0.5} \mathrm{Zr}_{0.5} \mathrm{O}_{2}, \mathrm{HZO}\right)$ and its thickness was $5 \mathrm{~nm}$. Figure $5 \mathrm{~b}, \mathrm{c}$ show the typical polarization versus voltage $(\mathrm{P}-\mathrm{V})$ curves and the endurance test result under successive voltage pulses with $2.5 \mathrm{~V}$. The $\mathrm{P}-\mathrm{V}$ loop showed a coercive voltage of $0.8 \mathrm{~V}$ and a remnant polarization $(\mathrm{Pr})$ of about $6.5 \mu \mathrm{C} / \mathrm{cm}^{2}$. The hysteresis curve shape became more antiferroelectric-like, and as the field circulated, it became ferroelectric-like. This phenomenon means that there are many antiferroelectric $\mathrm{t}$-phases in the pristine state of the thin film. With the electric field cycling, the antiferroelectric phases changed into ferroelectric o-phases, which is similar to previous reports [26-28]. As the electric field circulated further, the polarization decreased to about $4 \mu \mathrm{C} / \mathrm{cm}^{2}$ after $10^{8}$ cycles, which is a characteristic of fatigue. It has been suggested that fatigue may be due to transition to a non-ferroelectric m-phase [29]. After understanding the macroscopic electrical properties of the sample, we further conducted an in situ electrical experiment on the sample, and the schematic diagram of the in situ experiment is shown in Figure 5d. A diagram of the upper left corner shows the schematic of the DC voltage-time profile applied on the probe.
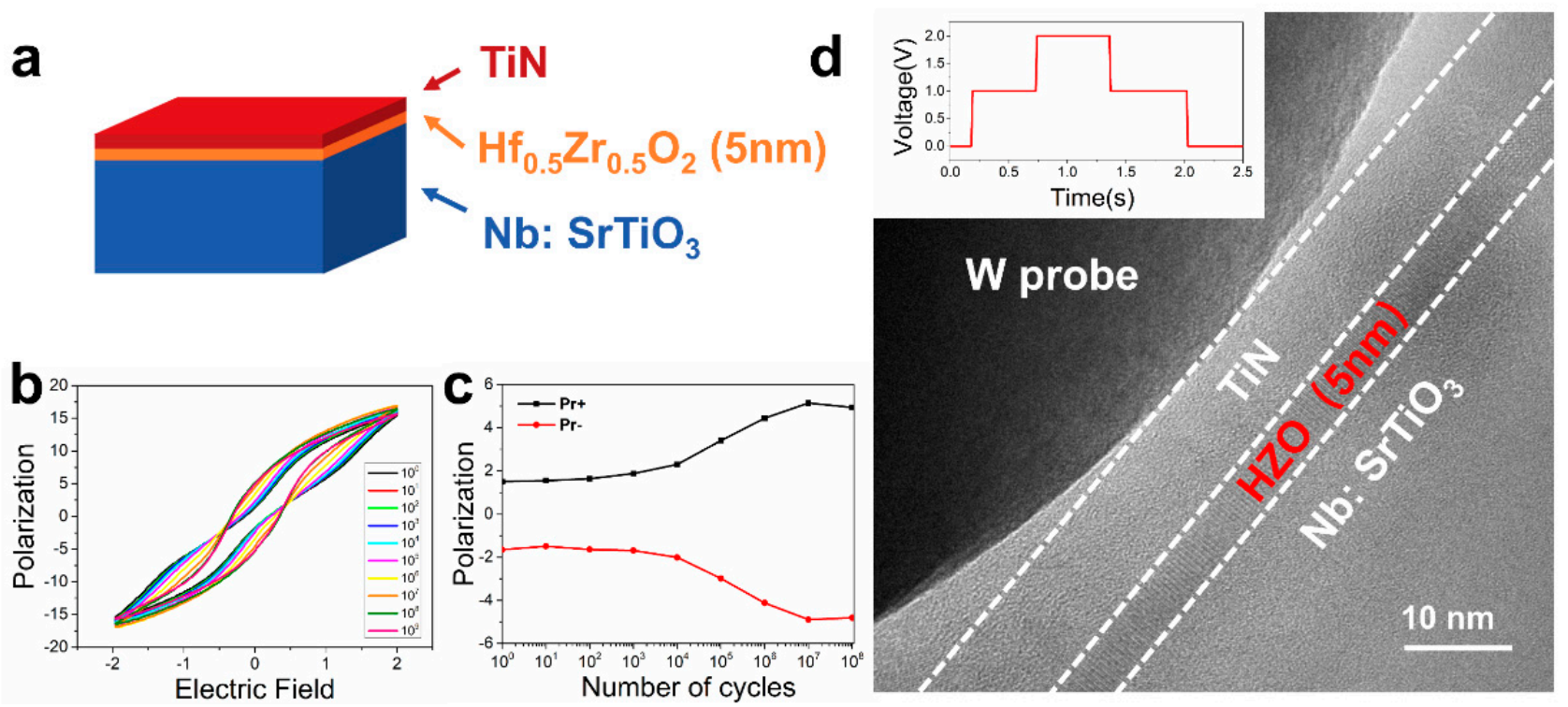

Figure 5. The $\mathrm{Hf}_{0.5} \mathrm{Zr}_{0.5} \mathrm{O}_{2}$ (HZO) thin film for in situ electrical experiments. (a) Schematic diagram of the HZO-based ferroelectric thin film capacitance structure for experiment. (b) Typical P-V curves of $\mathrm{HZO}(5 \mathrm{~nm})$ capacitor. (c) The tendency of Pr to cycles under 2.5 V. (d) High resolution TEM (HRTEM) image shows the in situ biasing process for a local area in the $\mathrm{HZO}$ thin film with the aid of tungsten probe.

Figure 6a shows the high magnification capacitor structure with a t-grain between the two electrodes projected along the T[110] direction. This grain was about $36 \mathrm{~nm}$ long and slightly trapezoidal. Figure $6 \mathrm{~b}$ contains enlarged high-angle annular dark-field (HAADF) and annular bright-field (ABF) images of the yellow boxes in Figure 6a. In this HAADF image, only heavy atoms such as $\mathrm{Hf}$ and $\mathrm{Zr}$ can be identified. In addition, the light element sensitive atomic resolution scanning transmission electron microscopy- annular bright-field (STEM-ABF) technique was used to detect oxygen atom information. The corresponding ABF experimental image of this grain shows oxygen in the middle of the heavy atoms. Figure $6 \mathrm{c}$ is the schematic of the HZO unit cell of the t-phase projected along the T[110] zone axes. The orange and red solid balls correspond to the $\mathrm{Hf} / \mathrm{Zr}$ and $\mathrm{O}$ atoms. The experimental and simulated results were completely consistent, as shown in the inset of the simulated result. The quality of this capacitor slice made by the above method is good 
and can be used in in situ electrical experiments. After the electric field cycle, the change of electrification process was very rapid, and there was a certain jitter in the electrification process. At the end of the cycle, the t-phase grain was found to have completely transformed into a m-phase grain. Figure $6 \mathrm{~d}$ shows the grain after electric field cycling, which was identified as an $\mathrm{m}$-phase grain along the M[100] direction. The shape and size of the grain hardly changed, and the phase structure of the grain changed as a whole. Figure 6e contains enlarged HAADF and ABF images of the yellow boxes in Figure $6 \mathrm{~d}$, and Figure $6 f$ is the schematic. The illustrations in the upper right corner of Figure $6 \mathrm{~b}, \mathrm{e}$ are the electron diffraction patterns obtained by using fast Fourier transformation (FFT). Structural change can be clearly observed from FFT change. The experimental results agree well with the simulation. The phase transformation process before and after the in situ electric field is consistent with the previous macroscopic properties, which proves that one of the causes of fatigue is the transformation of part of the antiferroelectric and ferroelectric phases to the non-ferroelectric phase. There has been much speculation in previous reports about the fatigue of hafnium-based ferroelectrics, the most recognized being the cause of the transition to the m-phase of non-ferroelectrics [29]. The transition from t-phase to o-phase has been verified theoretically and experimentally [30,31]. The direct current used in this experiment could cause a direct failure. In the actual sample, the process from awakening to fatigue appears, which is regarded as a transition from t to o to $\mathrm{m}$ phase.

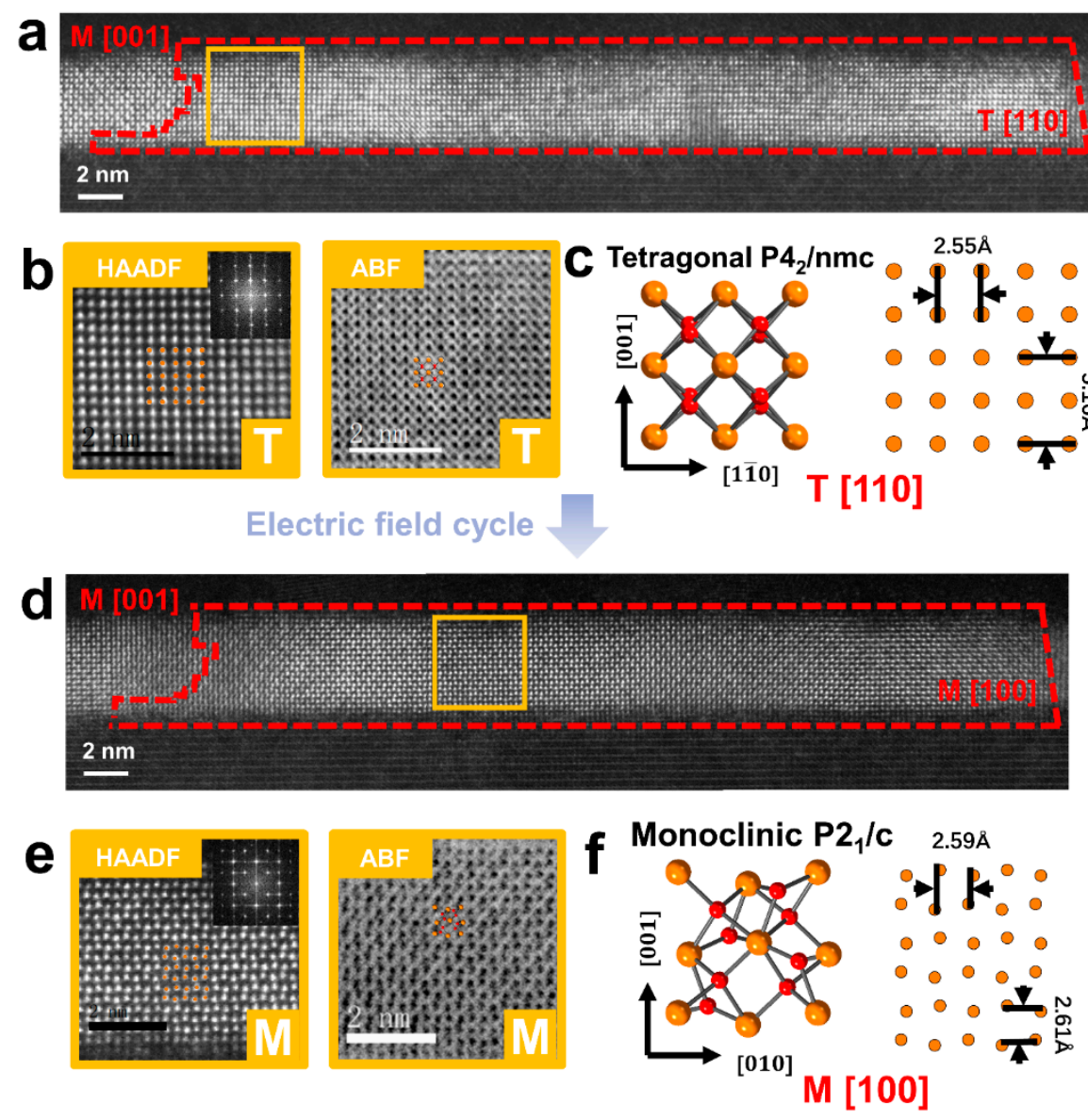

Figure 6. Atomic structure changes in phase transition region before and after electrification. (a) High magnification capacitor structure before electrification. (b) Enlarged high-angle annular dark-field (HAADF) and annular bright-field (ABF) diagrams of t-phases. (c) Atomic model of tetragonal phase. (d) High magnification capacitor structure after electrification. (e) Enlarged HAADF and ABF diagrams of m-phases monoclinic phase. (f) Atomic model of monoclinic phase. 
Figure 7 shows another case which the m-phase grain did not change under the action of electric field. Figure 7a shows the grain shape of $\mathrm{m}$-phase before and after electrification. Figure $7 \mathrm{~b}$ shows the atomic model in m-phase M[001] orientation, corresponding to the enlarged HAADF and ABF images in Figure 7c. It can be shown that m-phase was stable under the action of electric field, and m-phase transformation would not occur during the electric field cycle.

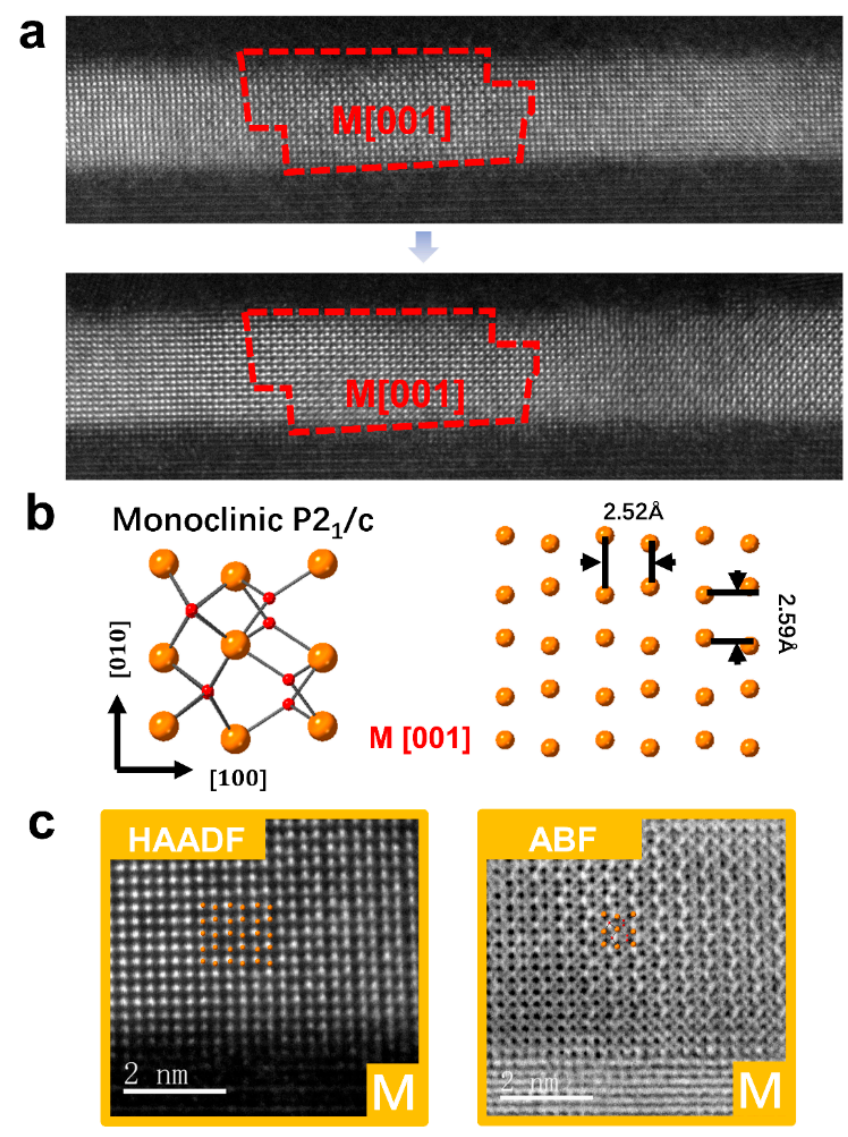

Figure 7. Regions where the phase structure did not change during electrification. (a) Low magnification image of grain that did not undergo phase transformation. (b) Atomic model of monoclinic phase. (c) Enlarged HAADF and ABF diagrams of m-phases.

\section{Discussion and Conclusions}

In this paper, we propose three methods to improve in situ sample preparation. Each method provides some improvement in sample preparation in three aspects: thin enough for better observation, more stable and more convenient for probe contact, and less leakage after contact. For the first point, the carbon layer positioning can help us to thin the sample to the upper electrode precisely, which is very effective for thin multilayer films, especially polycrystalline films. The sample prepared by FIB is wedge-shaped, with the thinnest at the front. With the positioning of the carbon protective layer, all the observed film areas can be thin, and the thickness of the sample will greatly affect the quality of observation. For the second point, welding the sample on the top of the B column provides the full sample range of the probe, so that the probe is not affected by the $\mathrm{Cu}$ grid. There are about 200 grains in the sample range of about 10 microns. The polycrystalline orientation is random, and the contact range of almost all grains can greatly improve the success rate. In addition, both sides of the bottom of the sample are welded to the $\mathrm{Cu}$ grid, providing a more stable support for the sample and reducing the deformation caused when the probe contacts the sample. Finally, cutting the sample into a comb shape is used to reduce leakage. When the capacitor is segmented, local leakage and other problems will not affect the whole sample. If the experiment of a single cut capacitor fails, we can still continue the experiment on the 
other cut capacitors, which further improves the success rate of a single experiment. In summary, we propound three tips to improve the success rate of in situ experiments. After the implementation of the above three tips, we successfully prepared HZO film samples that could be used for in situ experiments. The obtained knowledge would be beneficial for optimizing the in situ biasing TEM sample preparation method, and therefore may be applicable to (and hence can be considered in) other types of device samples, with much broader implications.

Author Contributions: Conceptualization: Y.C.; data curation: Q.Z. and Y.W.; formal analysis: Q.Z., Y.W., Y.Z. (Yunzhe Zheng), Y.Z. (Yonghui Zheng), Z.G. and T.X.; methodology: Y.C. and H.L.; project administration: Y.C. and H.L.; resources: Y.C., H.L. and R.H.; writing-original draft: Q.Z. and Y.W.; writing-review and editing: Y.C., Y.Z. (Yonghui Zheng), R.H. and H.L. All authors have read and agreed to the published version of the manuscript.

Funding: This work is supported by the National Key Research and Development Program of China (2017YFA0303403), the National Natural Science Foundation of China (92064003, 62174054, 62104071, 12134003, 61974042), the Strategic Priority Research Program of the Chinese Academy of Sciences under Grant No. XDB44010300, and the Shanghai Sailing Program (21YF1410900).

Acknowledgments: The authors thank Yue Liu from Shanghai Jiao Tong University for the in situ TEM biasing holder support.

Conflicts of Interest: The authors declare no conflict of interest.

\section{References}

1. Müller, J.; Polakowski, P.; Müller, S.; Mikolajick, T. Ferroelectric Hafnium Oxide Based Materials and Devices: Assessment of Current Status and Future Prospects. ECS Meet. Abstr. 2014, 4, N30-N35. [CrossRef]

2. Mikolajick, T.; Slesazeck, S.; Park, M.H.; Schroeder, U. Ferroelectric hafnium oxide for ferroelectric random-access memories and ferroelectric field-effect transistors. MRS Bull. 2018, 43, 340-346. [CrossRef]

3. Ota, K.; Deguchi, J.; Fujii, S.; Saitoh, M.; Yamaguchi, M.; Berdan, R.; Marukame, T.; Nishi, Y.; Matsuo, K.; Takahashi, K.; et al. Performance Maximization of In-Memory Reinforcement Learning with Variability-Controlled Hf1-xZrxO ${ }_{2}$ Ferroelectric Tunnel Junctions. In Proceedings of the 2019 IEEE International Electron Devices Meeting (IEDM), San Francisco, CA, USA, 7-11 December 2019. [CrossRef]

4. Zhao, B.; Jiang, J.; He, L.; Meng, J.; Geng, W.; Chen, Z.; Jiang, A. The effect of in situ annealing oxygen pressure on the ferro-electric resistive switching characteristic. Ceram. Int. 2015, 41, S835-S840. [CrossRef]

5. Park, M.H.; Chung, C.C.; Schenk, T.; Richter, C.; Opsomer, K.; Detavernier, C.; Adelmann, C.; Jones, J.L.; Mikolajick, T.; Schroeder, U. Effect of Annealing Ferroelectric $\mathrm{HfO}_{2}$ Thin Films: In Situ, High Temperature X-ray Diffraction. Adv. Electron. Mater. 2018, 4, 1800091. [CrossRef]

6. Park, M.H.; Lee, Y.H.; Kim, H.J.; Schenk, T.; Lee, W.; Kim, K.D.; Fengler, F.P.G.; Mikolajick, T.; Schroeder, U.; Hwang, C.S. Surface and grain boundary energy as the key enabler of ferroelectricity in nanoscale hafnia-zirconia: A comparison of model and experiment. Nanoscale 2017, 9, 9973-9986. [CrossRef]

7. Sang, X.; Grimley, E.D.; Schenk, T.; Schroeder, U.; Lebeau, J.M. On the structural origins of ferroelectricity in $\mathrm{HfO}_{2}$ thin films. Appl. Phys. Lett. 2015, 106, 162905. [CrossRef]

8. Park, M.H.; Lee, Y.H.; Kim, H.J.; Kim, Y.J.; Moon, T.; Kim, K.D.; Hyun, S.D.; Mikolajick, T.; Schroeder, U.; Hwang, C.S. Understanding the formation of the metastable ferroelectric phase in hafnia-zirconia solid solution thin films. Nanoscale 2017, 10, 716-725. [CrossRef] [PubMed]

9. Qi, Y.B.; Singh, S.; Lau, C.; Huang, F.T.; Xu, X.H.; Walker, F.J.; Ahn, C.H.; Cheong, S.W.; Rabe, K.M. Stabilization of Com-peting Ferroelectric Phases of $\mathrm{HfO}_{2}$ under Epitaxial Strain. Phys. Rev. Lett. 2020, 125, 257603. [CrossRef]

10. Dogan, M.; Gong, N.; Ma, T.-P.; Ismail-Beigi, S. Causes of ferroelectricity in $\mathrm{HfO}_{2}$-based thin films: An ab initio perspective. Phys. Chem. Chem. Phys. 2019, 21, 12150-12162. [CrossRef]

11. Fan, P.; Zhang, Y.K.; Yang, Q.; Jiang, J.; Jiang, L.M.; Liao, M.; Zhou, Y.C. Origin of the Intrinsic Ferroelectricity of $\mathrm{HfO}_{2}$ from ab Initio Molecular Dynamics. J. Phys. Chem. C 2019, 123, 21743-21750. [CrossRef]

12. Nukala, P.; Ahmadi, M.; Antoja-Lleonart, J.; de Graaf, S.; Wei, Y.; Zandbergen, H.W.; Kooi, B.J.; Noheda, B. In situ heating studies on temperature-induced phase transitions in epitaxial $\mathrm{Hf}_{0.5} \mathrm{Zr}_{0.5} \mathrm{O}_{2} / \mathrm{La}_{0.6} 7 \mathrm{Sr}_{0.33} \mathrm{MnO}_{3}$ heterostructures. Appl. Phys. Lett. 2021, 118, 062901. [CrossRef]

13. Nukala, P.; Ahmadi, M.; Wei, Y.; de Graaf, S.; Stylianidis, E.; Chakrabortty, T.; Matzen, S.; Zandbergen, H.W.; Björling, A.; Mannix, D.; et al. Reversible oxygen migration and phase transitions in hafnia-based ferroelectric devices. Science 2021, 372, 630-635. [CrossRef] [PubMed]

14. Kwon, D.-H.; Kim, K.M.; Jang, J.H.; Jeon, J.M.; Lee, M.H.; Kim, G.H.; Li, X.-S.; Park, G.-S.; Lee, B.; Han, S.; et al. Atomic structure of conducting nanofilaments in $\mathrm{TiO}_{2}$ resistive switching memory. Nat. Nanotechnol. 2010, 5, 148-153. [CrossRef] [PubMed] 
15. Liao, H.G.; Zherebetskyy, D.; Xin, H.L.; Czarnik, C.; Ercius, P.; Elmlund, H.; Pan, M.; Wang, L.W.; Zheng, H.M. Facet de-velopment during platinum nanocube growth. Science 2014, 345, 916-919. [CrossRef] [PubMed]

16. Jia, C.L.; Lentzen, M.; Urban, K. Atomic-Resolution Imaging of Oxygen in Perovskite Ceramics. Science 2003, $299,870-873$. [CrossRef]

17. Huang, R.; Ikuhara, Y. STEM characterization for lithium-ion battery cathode materials. Curr. Opin. Solid State Mater. Sci. 2012, 16, 31-38. [CrossRef]

18. Deng, Y.; Zhang, R.; Pekin, T.C.; Gammer, C.; Ciston, J.; Ercius, P.; Ophus, C.; Bustillo, K.; Song, C.; Zhao, S.; et al. Functional Materials Under Stress: In Situ TEM Observations of Structural Evolution. Adv. Mater. 2019, 32, e1906105. [CrossRef]

19. Narasimhan, V.K.; McBriarty, M.E.; Passarello, D.; Adinolfi, V.; Toney, M.F.; Mehta, A.; Littau, K.A. In Situ Characterization of Ferroelectric $\mathrm{HfO}_{2}$ During Rapid Thermal Annealing. Phys. Status Solidi (RRL) Rapid Res. Lett. 2021, 15, 2000598. [CrossRef]

20. Yu, Q.; Mao, M.-M.; Li, Q.-J.; Fu, X.-Q.; Tian, H.; Li, J.-X.; Mao, S.X.; Zhang, Z. In Situ Observation on Dislocation-Controlled Sublimation of Mg Nanoparticles. Nano Lett. 2016, 16, 1156-1160. [CrossRef]

21. Zhang, D.; Jin, C.; Tian, H.; Xiong, Y.; Zhang, H.; Qiao, P.; Fan, J.; Zhang, Z.; Li, Z.Y.; Li, J. An In situ TEM study of the surface oxidation of palladium nanocrystals assisted by electron irradiation. Nanoscale 2017, 9, 6327-6333. [CrossRef]

22. Si, J.; Liu, Y.; He, Z.; Du, H.; Du, K.; Chen, D.; Li, J.; Xu, M.; Tian, H.; He, H.; et al. Efficient and High-Color-Purity Light-Emitting Diodes Based on In Situ Grown Films of $\mathrm{CsPbX}_{3}(\mathrm{X}=\mathrm{Br}$, I) Nanoplates with Controlled Thicknesses. ACS Nano 2017, 11, 11100-11107. [CrossRef] [PubMed]

23. Yang, X.; Luo, C.; Tian, X.; Liang, F.; Xia, Y.; Chen, X.; Wang, C.; Liang, S.X.; Wu, X.; Chu, J. A revew of in situ transmission electron microscopy study on the switching mechanism and packaging reliability in non-volatile memory. J. Semicond. 2021, 42. [CrossRef]

24. Yang, Y.; Lu, W.; Yao, Y.; Sun, J.; Gu, C.; Gu, L.; Wang, Y.; Duan, X.; Yu, R. In situ TEM Observation of Resistance Switching in Titanate Based Device. Sci. Rep. 2014, 4, 3890. [CrossRef] [PubMed]

25. Gao, P.; Wang, Z.; Fu, W.; Liao, Z.; Liu, K.; Wang, W.; Bai, X.; Wang, E. In situ TEM studies of oxygen vacancy migration for electrically induced resistance change effect in cerium oxides. Micron 2010, 41, 301-305. [CrossRef]

26. Park, M.H.; Kim, H.J.; Kim, Y.J.; Lee, W.; Moon, T.; Hwang, C.S. Evolution of phases and ferroelectric properties of thin $\mathrm{Hf}_{0.5} \mathrm{Zr}_{0.5} \mathrm{O}_{2}$ films according to the thickness and annealing temperature. Appl. Phys. Lett. 2013, 102, 242905. [CrossRef]

27. Park, M.H.; Kim, H.J.; Kim, Y.J.; Lee, W.; Moon, T.; Kim, K.D.; Hwang, C.S. Study on the degradation mechanism of the ferroelectric properties of thin $\mathrm{Hf}_{0.5} \mathrm{Zr}_{0.5} \mathrm{O}_{2}$ films on TiN and Ir electrodes. Appl. Phys. Lett. 2014, 105, 072902. [CrossRef]

28. Park, M.H.; Kim, H.J.; Kim, Y.J.; Lee, Y.H.; Moon, T.; Kim, K.D.; Hyun, S.D.; Hwang, C.S. Study on the size effect in $\mathrm{Hf}_{0.5} \mathrm{Zr}_{0.5} \mathrm{O}_{2}$ films thinner than $8 \mathrm{~nm}$ before and after wake-up field cycling. Appl. Phys. Lett. 2015, 107, 192907. [CrossRef]

29. Fields, S.S.; Smith, S.W.; Ryan, P.J.; Jaszewski, S.; Brummel, I.A.; Salanova, A.; Esteves, G.; Wolfley, S.L.; Henry, M.D.; Davids, P.S.; et al. Phase-Exchange-Driven Wake-Up and Fatigue in Ferroelectric Hafnium Zirconium Oxide Films. ACS Appl. Mater. Interfaces 2020, 12, 26577-26585. [CrossRef]

30. Liu, S.; Hanrahan, B.M. Effects of growth orientations and epitaxial strains on phase stability of $\mathrm{HfO}_{2}$ thin films. Phys. Rev. Mater. 2019, 3, 054404. [CrossRef]

31. Zheng, Y.; Zhong, C.; Zheng, Y.; Gao, Z.; Cheng, Y.; Zhong, Q.; Liu, C.; Wang, Y.; Qi, R.; Huang, R.; et al. In-situ atomic visualization of structural transformation in $\mathrm{Hf}_{0.5} \mathrm{Zr}_{0.5} \mathrm{O}_{2}$ ferroelectric thin film: From nonpolar tetragonal phase to polar orthorhombic phase. In Proceedings of the 2021 Symposium on VLSI Technology, Kyoto, Japan, 13-19 June 2021; pp. 1-2. 